\title{
Presença de Listeria monocytogenes em Queijos de Pasta Mole da Região a Sul do Tejo
}

\author{
Magda Gonçalves ${ }^{a}$ Rosália Furtado $^{b}$ Anabela Coelho $^{b}$ Cristina Belo Correia ${ }^{b}$ \\ Ana Valente ${ }^{a, c}$ \\ aCentro de Estudos Sociedade, Organizações e Bem Estar, Universidade Atlântica, Barcarena, Portugal; \\ ${ }^{b}$ Departamento de Alimentação e Nutrição, Instituto Nacional de Saúde Doutor Ricardo Jorge, I.P. (INSA), Lisbon, \\ Portugal; ' Instituto de Saúde Ambiental (ISAMB), Faculdade de Medicina, Universidade de Lisboa, Lisbon, Portugal
}

\begin{abstract}
Palavras Chave
Listeria monocytogenes · Listeriose · Queijo · Saúde pública · Toxinfeção
\end{abstract}

\section{Resumo}

Introdução: Nas últimas décadas, o número de casos de infeções de origem alimentar provocadas por Listeria monocytogenes tem vindo a aumentar. Objetivo: Deteção e quantificação de Listeria monocytogenes em queijos de pasta mole produzidos na região a Sul do Tejo. Metodologia: Foram analisadas 30 amostras de queijos de pasta mole de diferentes fabricantes, dos quais $66,7 \%$ produzidas a partir de leite de ovelha cru. Resultados: Das amostras analisadas, 10\% $(n=3)$ estavam contaminadas com Listeria monocytogenes e $17 \%(n=5)$ com Listeria innocua. Conclusão: O consumo de queijos de pasta mole está associado com o aumento do risco de ocorrência de listeriose.

(c) 2017 The Author(s). Published by S. Karger AG, Basel on behalf of Escola Nacional de Saúde Pública

\section{The Presence of Listeria monocytogenes in Soft Cheeses from the Southern Region of Tejo}

\section{Keywords}

Listeria monocytogenes · Listeriosis - Cheese · Public health · Foodborne outbreak

\begin{abstract}
Introduction: In the past decades, the number of food poisoning outbreaks brought on by Listeria monocytogenes has been increasing. Methods: We analyzed 30 samples of soft cheeses from different manufacturers, of which $66.7 \%$ were produced from raw sheep milk. $\boldsymbol{R e}$ sults: The presence of $L$. monocytogenes was detected in $10 \%(n=3)$ of the analyzed samples, and $17 \%(n=5)$ were contaminated with L. innocua. Conclusion: The consumption of soft cheeses is associated with an increased risk of listeriosis.

C 2017 The Author(s). Published by S. Karger AG, Basel on behalf of Escola Nacional de Saúde Pública
\end{abstract}

\section{KARGER}

E-Mail karger@karger.com www.karger.com/pjp (c) 2017 The Author(s). Published by S. Karger AG, Basel on behalf of Escola Nacional de Saúde Pública

Karcer

0 pen eccess

This article is licensed under the Creative Commons AttributionNonCommercial-NoDerivatives 4.0 International License (CC BYNC-ND) (http://www.karger.com/Services/OpenAccessLicense) Usage and distribution for commercial purposes as well as any distribution of modified material requires written permission.
Ana Valente, $\mathrm{PhD}$

Departamento de Ciências da Nutrição Universidade Atlântica

PT-2730-036 Barcarena (Portugal)

E-Mail avalente@ uatlantica.pt 


\section{Introdução}

A contaminação microbiológica dos alimentos pode contribuir para a transmissão de doenças de origem alimentar, as quais representam um problema de saúde com grande impacto a nível mundial [1].

Os patogénicos de origem alimentar são microrganismos capazes de infetar os seres humanos pelo consumo de alimentos e águas contaminados, provocando diferentes sintomas [2-4] (ex. náuseas, vómitos, diarreia, mal-estar, febre e dores abdominais) que em alguns casos podem evoluir e originar sequelas mais graves por vezes fatais. As toxinfeções alimentares são um grupo de doenças de origem alimentar que incluem: (1) infeções alimentares: causadas pela ingestão de agentes infeciosos vivos; (2) intoxicações alimentares: provocadas pela ingestão de toxinas microbianas formadas nos alimentos antes do seu consumo; (3) toxinfeções: originadas pela ingestão de um alimento que contenha microorganismos patogénicos, que produzem toxinas dentro dos intestinos [2].

As bactérias mais frequentemente reportadas como causadoras de doenças de origem alimentar são a Salmonella enterica, Staphylococcus aureus, Escherichia coli, Listeria monocytogenes, Campylobacter spp. e Bacillus cereus [5].

Devido à severidade e elevada taxa de mortalidade da listeriose humana [5], é fundamental em saúde pública a deteção de Listeria monocytogenes em alimentos prontos para consumo, nomeadamente em queijos de pasta mole, dado estarem descritos surtos e casos esporádicos de listeriose associados a este tipo de produtos, mantidos em condições de refrigeração e durante um determinado período de vida útil.

A Listeria monocytogenes é uma bactéria Gram-positiva com a capacidade de se multiplicar em ambientes adversos. É conhecida e estudada desde 1924 por Murray et al. [6], sendo a única espécie reconhecida do género Listeria até cerca de 1961. Estão atualmente estudadas 10 espécies: L. monocytogenes, L. innocua, L. welshimeri, $L$. seeligeri, L. grayi e L. ivanovii e mais recentemente L. marthii, L. rocourtiae, L. fleischmannii, e L. weihenstephanensis, sendo os casos de infeção em humanos quase exclusivamente causados pela espécie L. monocytogenes [1].

Esta espécie pode originar quadros clínicos de septicemia, gastroenterite, meningite e encefalite. Os grupos de maior risco incluem crianças, indivíduos imunodeprimidos, grávidas e idosos [7]. Segundo Mylonakis et al. [8] e Lamont et al. [9], durante a gravidez a infeção com Listeria monocytogenes pode ter consequências graves, incluindo aborto, morte fetal e materna, parto prematuro, e sépsis neonatal [10]. A evolução da doença depende principalmente da quantidade de bactérias ingeridas, da virulência da estirpe e da suscetibilidade do hospedeiro. Deste modo, é difícil determinar a dose infeciosa mínima de patogénico para provocar doença $[10,11]$. Numa fase inicial os sintomas são semelhantes aos manifestados em outras gastroenterites de origem alimentar (listeriose não invasiva). No entanto a capacidade para atravessar barreiras fisiológicas como a placenta, o intestino e a barreira hematoencefálica aumenta a severidade da doença (listeriose invasiva).

O período de latência da doença pode ser longo, estando descrito por diferentes autores um período médio de 8 dias para a listeriose invasiva mas podendo variar de 1 a 67 dias $[12,13]$.

Esta bactéria pode estar presente no meio ambiente, sendo transmitida principalmente pela via alimentar. Diferentes tipos de alimentos têm sido associados a surtos de listeriose, como carnes, peixes fumados, saladas, leite e produtos derivados não pasteurizados nomeadamente queijos de pasta mole, vegetais, produtos de charcutaria, alimentos prontos a comer e alimentos processados [1, 14]. Para além dos alimentos poderem ser fonte de contaminação também o ambiente de processamento (utensílios utilizados na sua preparação e os equipamentos associados ao fabrico), pode ser um veículo de contaminação se a higienização e desinfeção não forem eficazes [15]. A Listeria monocytogenes apresenta grande capacidade para se alojar em locais onde é difícil a penetração dos detergentes e desinfetantes, aderindoàs superfícies dos equipamentos e formando biofilmes que as protegem de condições ambientais adversas [16].

A prevenção da listeriose pode ter diferentes abordagens: (a) cozinhar bem alimentos crus de origem animal (ex. carnes bovinas, suínas e de aves); (b) lavar os vegetais crus completamente em água corrente antes de comer, separar alimentos cozinhados de alimentos crus; (c) não beber leite cru e não comer alimentos que têm leite não pasteurizado neles; (d) lavar as mãos, facas, bancadas, e tábuas de corte após o manuseamento e preparação de alimentos não cozidos; (e) consumir alimentos perecíveis e prontos-a-comer o mais rapidamente possível. Nos Estados Unidos da América adotou-se uma política de tolerância zero para a presença de Listeria monocytogenes nos alimentos prontos para consumo. Na Europa, segundo o Regulamento (CE) no 2073/2005 [17], é permitida a presença de Listeria monocytogenes até ao limite de $100 \mathrm{ufc} / \mathrm{g}$ em alimentos prontos para consumo (onde se incluem os queijos de pasta mole) colocados no mercado durante o seu período de vida útil. No fim do processo de fabrico
38

Port J Public Health 2017;35:37-43 DOI: $10.1159 / 000477648$
Gonçalves/Furtado/Coelho/Correia/ Valente 
Table 1. Caracterização das amostras em estudo

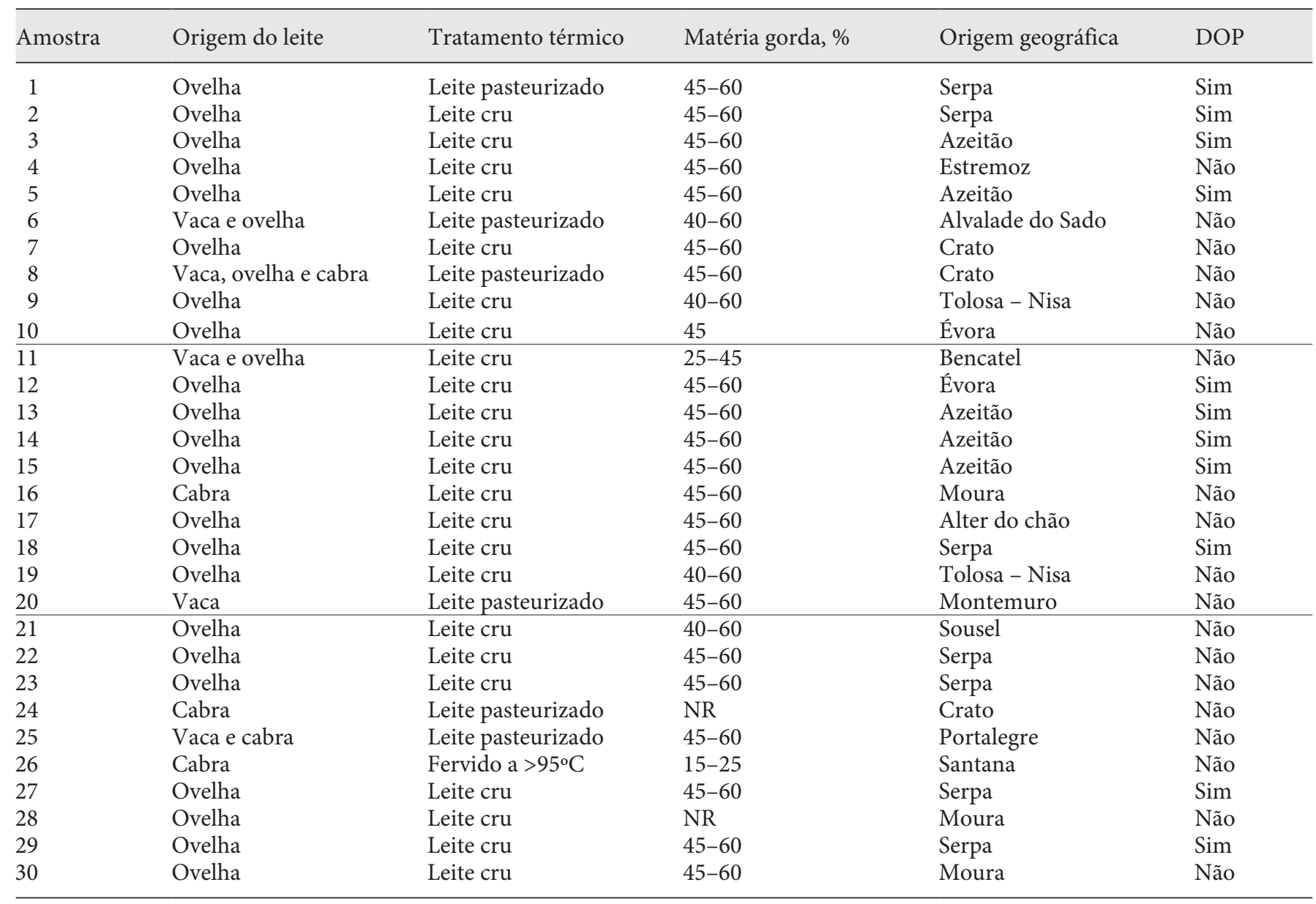

DOP, Denominação de Origem Protegida; NR, não referido.

dos alimentos suscetíveis de permitir o crescimento de Listeria monocytogenes, é exigida a ausência de Listeria monocytogenes em $25 \mathrm{~g}$ no momento que o alimento deixa de estar sob o controlo do operador da empresa do setor alimentar que o produziu. A exceção ocorre quando o produtor tem a capacidade para demonstrar que o produto não excederá o limite de $100 \mathrm{ufc/g}$ até ao fim do seu período de vida útil.

A notificação de listeriose em humanos é obrigatória na maioria dos Estados Membros. Em Portugal esta doença é de declaração obrigatória [18] desde abril de 2014, no entanto os dados disponíveis no que se refere ao número de surtos são muito reduzidos a nível nacional. Em 2009, Almeida et al. [19] publicaram um artigo onde descrevem todos os casos diagnosticados em Portugal no período de 2004 a 2007. Identificaram-se 67 casos, sendo que dos 24 casos para os quais foi conhecida a evolução clínica, $40 \%$ foram fatais [19]. No ano de 2007 em Portugal, estimouse uma taxa de incidência de 2,3 casos por milhão de habitantes, sendo este valor semelhante à taxa de incidência verificada na Áustria, Estónia, Letónia, Eslováquia e Eslovénia [20].

Em Portugal existe uma forte tradição no consumo de produtos regionais de charcutaria, nomeadamente de queijos de pasta mole. Este alimento pelas matérias primas utilizadas (ex. leite cru) ou durante o processo de fabrico pode ser um veículo de contaminação de Listeria monocytogenes se a higienização e desinfeção não forem eficazes. O presente estudo teve como objetivo a deteção e quantificação de Listeria monocytogenes em queijos de pasta mole produzidos na região a Sul do Tejo, uma das regiões portuguesas com tradição na produção deste tipo de queijos. 


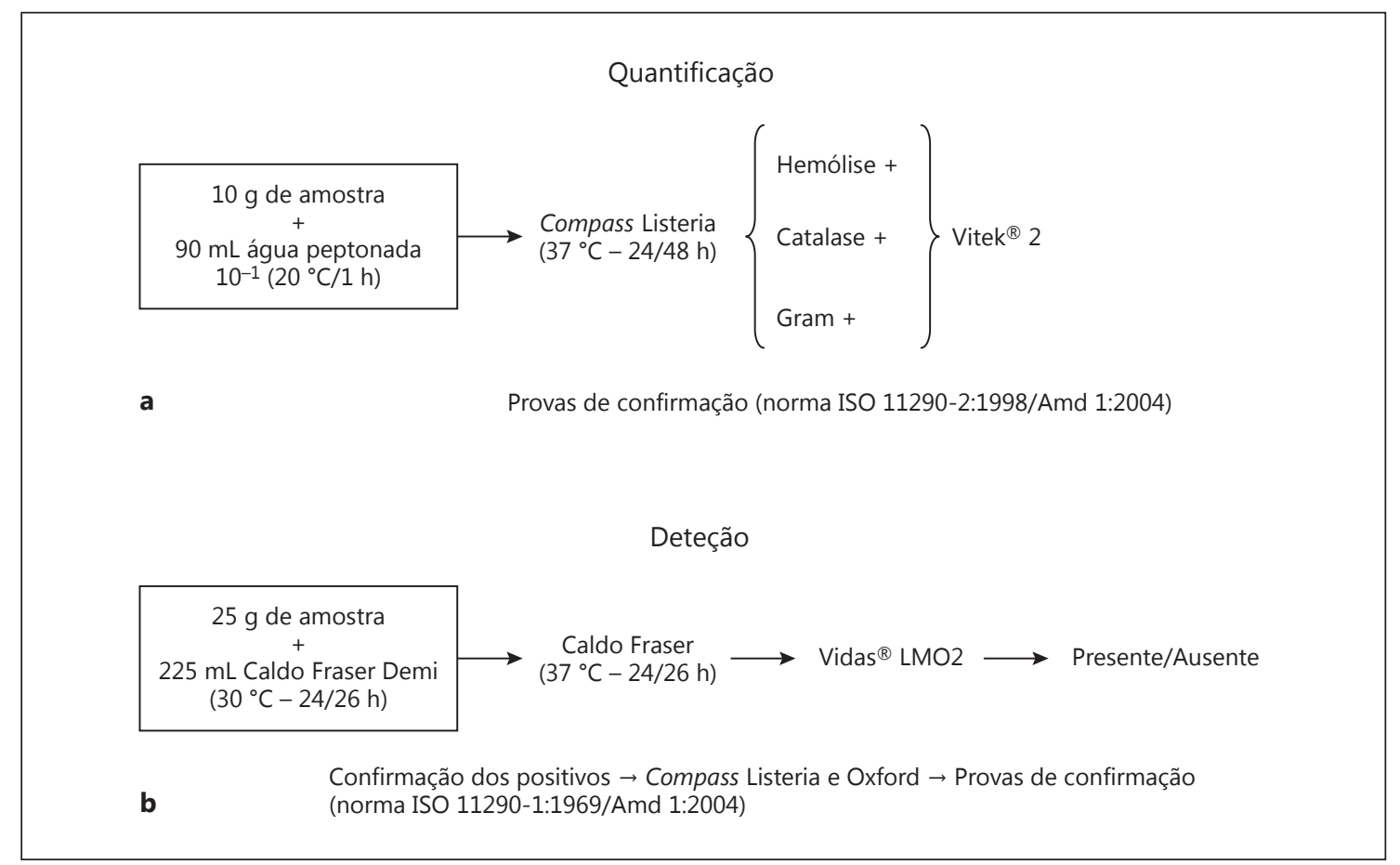

Fig. 1. Procedimentos. a Contagem de Listeria spp. segundo o método normativo ISO 11290-2:1998/Amd 1:2004. b Pesquisa de Listeria monocytogenes pelo método VIDAS ${ }^{\circledR}$ LMO2.

\section{Materiais e Métodos}

\section{Amostragem}

No presente trabalho foram analisadas 30 amostras de queijo de diferentes fabricantes, adquiridos em seis grandes superfícies comerciais da região de Lisboa. As amostras foram recolhidas entre os meses de março e abril de 2014 e transportadas em mala isotérmica para o Laboratório de Microbiologia do Departamento de Alimentação e Nutrição (DAN) do Instituto Nacional de Saúde Doutor Ricardo Jorge, I.P. (INSA). Todos os queijos foram devidamente identificados e codificados de acordo com a origem do leite, tratamento térmico, matéria gorda e origem geográfica (Tabela 1).

\section{Colheita e Preparação da Amostra}

As amostras foram recolhidas seguindo regras de assepsia numa câmara de fluxo laminar Vertical classe ISO5 marca Microflow $^{\circledR}$ e modelo 20134 (Astec, Andover, UK). O exterior de todas as amostras embaladas, foi desinfetado com etanol a $70 \%(\mathrm{v} / \mathrm{v})$ e efetuada a colheita de uma porção de cerca de $100 \mathrm{~g}$ para sacos estéreis identificados de 1 a 30. Cada amostra foi recolhida numa proporção de $10 \%$ de casca e $90 \%$ de massa. De seguida foram efetuadas as diluições iniciais da amostra (Fig. 1) em ambiente controlado e com material estéril. Depois, as amostras foram homogeneizadas durante $1 \mathrm{~min}$ a $230 \mathrm{rpm}$, num agitador peristáltico da marca Stomacher ${ }^{\circledR}$, modelo 400 Circulator (Seward Limited, London, UK).
Contagem de Listeria spp.

Foi utilizado um método normativo [21, 22] para a contagem de Listeria spp. (incluindo Listeria monocytogenes) estando o referido procedimento descrito na Figura 1a. A diluição inicial da amostra $\left(1 \times 10^{-1}\right)$ foi efetuada com água peptonada tamponada, a partir de uma toma de $10 \mathrm{~g}$ utilizando um diluidor automático marca IUL Instruments, modelo Pinch Diluter, (Seward Limited, London, UK). A amostra foi colocada a $20 \pm 2{ }^{\circ} \mathrm{C}$ durante 1 hora em banho de água da marca Grant, modelo OLS200 (Grant Instruments, Cambridge, UK). O meio de cultura utilizado para a quantificação foi o meio seletivo COMPASS Listeria agar, (Biokar ${ }^{\circledR}$, Beauvais, France). Neste meio as bactérias do género Listeria spp., crescem desenvolvendo colónias azuis esverdeadas pela ação da enzima $\beta$-glucosidase sobre o X-glucosido. Para efetuar a identificação de Listeria spp. e de Listeria monocytogenes selecionaram-se 5 colónias características de cada placa contável.

\section{Identificação de Listeria spp.}

Para a identificação das estirpes de Listeria, realizaram-se as provas da catalase, coloração de Gram e hemólise a partir das colónias suspeitas obtidas nos meios seletivos. A identificação bioquímica das estirpes de Listeria foi efetuada no equipamento da marca Vitek ${ }^{\circledR}$ (bioMérieux ${ }^{\circledR} 185$ SA, Marcy l'Étoile, France), sistema automatizado para identificação bacteriana.

\section{Pesquisa de Listeria Monocytogenes}

Para a pesquisa de Listeria monocytogenes foi utilizado o método Vidas ${ }^{\circledR}$ LMO2, sendo a confirmação efetuada por um método
Gonçalves/Furtado/Coelho/Correia/ Valente 
normativo [22, 23]. Na Figura 1b está esquematizado o procedimento de pesquisa de Listeria monocytogenes. Para efetuar esta deteção, foram pesadas $25 \mathrm{~g}$ de amostra numa balança digital da marca Mettler Toledo, modelo PM 2000 (Mettler-Toledo AG, Laboratory and Weighing Technologies, Greifensee, Switzerland) e adicionadas de $225 \mathrm{~mL}$ Caldo Fraser meia concentração, seguido de um novo enriquecimento em Caldo Fraser. Após a etapa de enriquecimento, a deteção foi efetuada por um método imunoenzimático, no sistema automatizado Vidas ${ }^{\circledR}$ LMO2 (bioMérieux ${ }^{\circledR}$ SA, Marcy l'Étoile, France), tecnologia Enzyme Linked Fluorescent Assay (ELFA). Nas amostras com resultados positivos efetuaramse provas de confirmação no sistema Vidas ${ }^{\circledR}$ de acordo com o procedimento referido anteriormente e descrito na norma ISO 11290 1:1996/Amd 2004.

\section{Resultados e Discussão}

O leite de ovelha sem tratamento térmico representou cerca de $70 \%(n=21)$ da matéria-prima utilizada na produção dos queijos analisados (Tabela 1), seguido do leite de mistura pasteurizado. Dos 30 queijos de pasta mole estudados, nove apresentaram Listeria spp., e destes, quatro apresentaram Listeria monocytogenes. Das amostras com Listeria monocytogenes, três apresentaram níveis superiores ao valor máximo admissível [24] (100 ufc/g) e uma inferior ao Limite de Deteção (10 ucf/g) do método de contagem (Tabela 2).

Três dos queijos positivos para Listeria monocytogenes provinham de leite de ovelha cru, sendo que um deles evidenciou a presença de três diferentes espécies de Listeria (L. monocytogenes, L. innocua e L. welshimeri) em simultâneo (Tabela 2). Em cinco dos 30 queijos analisados foi detetada a espécie Listeria innocua (Tabela 2), considerada por alguns autores $[25,26]$ como indicador da possível presença de Listeria monocytogenes, contudo esta estirpe não foi detetada nestas amostras. Num único queijo de cabra, cujo leite sofreu tratamento térmico elevado (cerca de $95^{\circ} \mathrm{C}$ ), foi também detetada a presença de Listeria innocua. Embora estejam disponíveis dados sobre a prevalência de Listeria monocytogenes em queijos de pasta mole [5], são poucos os estudos realizados na Europa que têm como objetivo principal detetar e quantificar a Listeria monocytogenes neste tipo de queijos. Ao comparar os resultados obtidos com os encontrados na literatura científica para queijos de pasta mole, verificou-se que num estudo publicado em 2012, cujo objetivo foi a determinação da prevalência de Listeria em queijos de pasta mole provenientes de diferentes produtores no País Basco, os resultados foram negativos para Listeria monocytogenes em 51 amostras de queijos analisadas. No entanto, 9,8\% dos queijos apresentaram resultados positivos para Listeria
Table 2. Queijos com resultados positivos para Listeria monocytogenes e outras espécies de Listeria

\begin{tabular}{|c|c|c|c|}
\hline Amostra & $\begin{array}{l}\text { Contagem de } \\
\text { Listeria } \\
\text { monocytogenes } \\
\text { (ufc/g) }\end{array}$ & $\begin{array}{l}\text { Pesquisa de } \\
\text { Listeria } \\
\text { monocytogenes }\end{array}$ & Espécie de Listeria \\
\hline 2 & $1,2 \times 10^{2 *}$ & Presente & L. monocytogenes \\
\hline 9 & $1,1 \times 10^{2 *}$ & Presente & L. monocytogenes \\
\hline 10 & $4,3 \times 10^{2 *}$ & Presente & $\begin{array}{l}\text { L monocytogenes, } \\
\text { L innocua e } \\
\text { L.welshimerii }\end{array}$ \\
\hline 11 & $<1 \times 10^{1 * *}$ & Presente & L.monocytogenes \\
\hline 12 & $<1 \times 10^{1 * *}$ & Ausente & L. innocua \\
\hline 13 & $<1 \times 10^{1 * *}$ & Ausente & L. innocua \\
\hline 14 & $<1 \times 10^{1 * *}$ & Ausente & L. innocua \\
\hline 15 & $<1 \times 10^{1 * *}$ & Ausente & L. innocua \\
\hline 26 & $<1 \times 10^{1 * *}$ & Ausente & L. innocua \\
\hline
\end{tabular}

* Superior ao Valor Máximo Admissível [25]. ** Inferior ao limite de deteção $\left(1 \times 10^{1} \mathrm{ufc} / \mathrm{g}\right)$.

spp., não sendo identificada a espécie de Listeria que estava na origem dessa percentagem [27].

Num outro estudo, realizado em 2011 em Itália, foram analisados 50 queijos de pasta mole, em que não foi detetada a presença de Listeria monocytogenes em nenhuma das amostras [28]. Em 2013 no Japão devido ao crescente consumo de queijo observado naquele país, foi efetuado um estudo em 126 queijos, 33 dos quais de pasta mole mas em nenhum foi detetada a presença de Listeria monocytogenes [29].

Nas amostras de queijo do presente estudo em que foi detetada a presença de Listeria monocytogenes, as causas da contaminação podem ser várias nomeadamente a origem do leite utilizado, o processo de fabrico (ex. a recontaminação após tratamento térmico do produto, tratamento térmico insuficiente e condições de armazenamento), a distribuição ou a comercialização. É de salientar que dos três queijos em que foi detetada a presença de Listeria monocytogenes acima do valor máximo admissível [23], um era um produto de Denominação de Origem Protegida. Num dos queijos fabricado com leite tratado termicamente, foi detetada Listeria innocua, o que alerta para a importância do controlo de outros pontos críticos no fabrico dos queijos, para além do tratamento térmico do leite para minimizar/eliminar a contaminação por Listeria monocytogenes. De acordo com o dados publicados no relatório do Centers for Disease Control and Prevention (CDC) [30], os surtos atribuídos a queijos entre 1998 
e 2011 ocorreram devido tanto à utilização de leite não pasteurizado para o fabrico dos queijos, bem como pela contaminação pós-pasteurização. No entanto, os queijos de pasta mole produzidos a partir de leite não pasteurizado estão associados a um risco 50 a 160 vezes superior de ter Listeria monocytogenes do que os produzidos a partir de leite pasteurizado [31].

Os resultados deste estudo estão em conformidade com as conclusões dos relatórios do CDC que alertam para a importância da implementação de sistemas de segurança alimentar, contribuindo assim para a prevenção da ocorrência de listeriose nos consumidores deste tipo de produtos (especialmente em grupos e risco), nomeadamente através da utilização de leite pasteurizado, implementação de um sistema de Hazard Analysis and Critical Control Point (HACCP) que diminua os riscos associados à contaminação pós-processamento como no manuseamento e corte dos queijos.

Este estudo teve como principal limitação a baixa representatividade da amostragem. De futuro pretende-se adquirir queijos não apenas em grandes superfícies comerciais, mas também diretamente ao fabricante para a realização de um estudo comparativo e verificar se os produtores das marcas dos queijos contaminados com Listeria spp. possuem sistemas de segurança alimentar implementados e efetivos.

\section{Conclusão}

Os queijos podem ser veículos potenciais de Listeria monocytogenes e constituir um risco para o consumidor. De acordo com o padrão encontrado, os queijos de pasta mole produzidos na região do Sul do Tejo contaminados com Listeria monocytogenes foram produzidos a partir de leite sem tratamento térmico. Estes resultados evidenciam que o consumo deste tipo de produtos está associado a um aumento da probabilidade de ocorrência de listeriose, especialmente nos grupos mais vulneráveis. O incumprimento das Boas Práticas de Higiene no fabrico, distribuição e comercialização dos queijos aumenta o risco da presença de Listeria monocytogenes neste tipo de produtos. A segurança alimentar dos queijos de pasta mole pode ser garantida através da aplicação de Boas Práticas de Higiene e da utilização de combinações adequadas dos diferentes factores de segurança alimentar como sendo, o pH, a atividade da água, concentração de sal e condições de tempo/temperatura ao longo do processo.

\section{Agradecimentos}

Os autores agradecem à Dra. Maria Antónia Calhau, Coordenadora do Departamento de Alimentação e Nutrição (DAN) no INSA por autorizar a realização deste trabalho de investigação aplicada e a toda a equipa do laboratório pela colaboração prestada durante o desenvolvimento do presente estudo.

\section{References}

1 European Food Safety Authority: Analysis of the baseline survey on the prevalence of Listeria monocytogenes in certain ready-to-eat foods in the EU, 2010-2011. Part A: Listeria monocytogenes prevalence estimates. EFSA J 2013;11:3241.

2 Dwivedi HP, Jaykus LA: Detection of pathogens in foods: the current state-of-the-art and future directions. Crit Rev Microbiol 2011; $37: 40-63$.

3 Aygun O, Pehlivanlar S: Listeria spp. in the raw milk and dairy products in Antakya. Food Control 2006;17:676-679.

4 Meyer-Broseta S, Diot A, Bastian S: Estimation of low bacterial concentration: Listeria monocytogenes in raw milk. Int J Food Microbiol 2003;80:1-15.

5 European Food Safety Authority (EFSA): European Centre for Disease Prevention and Control (ECDC). The European Union Summary Report on Trends and Sources of Zoonoses, Zoonotic Agents and Food-Borne Outbreaks in 2012. EFSA J 2014;12:3547.
6 Murray EGD, Webb RA, Swan MBRA: A disease of rabbits characterised by a large mononuclear leukocytosis caused by a hitherto undescribed bacillus Bacterium monocyotogenes. J Pathol Bacteriol 1926;29:407-439.

7 Wiedmann M: Molecular subtyping methods for Listeria monocytogenes. JAOAC Int 2002; 85:524-531.

8 Mylonakis E, Paliou M, Hohmann EL, Calderwood SB, Wing EJ: Listeriosis during pregnancy: a case series and review of 222 cases. Medicine 2002;81:260-269.

9 Lamont RF, Sobel J, Mazaki-Tovi S, Kusanovic JP, Vaisbuch E, Kim SK, et al: Listeriosis in human pregnancy: a systematic review. J Perinat Med 2011;39:227-236.

10 Vázquez J, Kunh M, Berche P, Chakraborty T, Dominguez-Bernal G, Goebel W, et al: Listeria pathogenesis and molecular virulence determinants. Clin Microbiol Rev 2001;14:584640.

11 Swaminathan B, Gerner-Smidt P: The epidemiology of human listeriosis. Microbes Infect 2007;9:1236-1243.
12 Goulet V, King LA, Vaillant V, Valk H: What is the incubation period for listeriosis? BMC Infect Dis 2013;13:2-7.

13 Linnan M, Mascola L, Lou XD, Goulet V, May $\mathrm{S}$, Salminen C, et al: Epidemic listeriosis associated with Mexican-style cheese. N Engl J Med 1988;319:823-828.

14 Garrido V, Vitas AI, García I: Survey of Listeria monocytogenes in ready-to-eat products: prevalence by brands and retail establishments for exposure assessment of listeriosis in Northern Spain. Food Control 2009;20:986991.

15 Lianou A, Sofos JN: A review of the incidence and transmission of Listeria monocytogenes in ready-to-eat products in retail and food service environments. J Food Protect 2007; 9:2004-2217.

16 Lado BH, Yousef AE: Listeriosis in humans; in Ryser ET, Marth EH (eds): Listeria, Listeriosis and Food Safety. New York, CRC Press, 2007, pp 283-304. 
17 Comissão das Comunidades Europeias: Regulamento (CE) no 2073 relativo aos critérios microbiológicos aplicáveis aos géneros alimentícios. JO 2005;L338:1-26.

18 Despacho no 5681-A/2014. DR. Série II. Suplemento. 82 (2014-04-29) 1374(12)11374(13). Notificação obrigatória de doenças transmissíveis e outros riscos em saúde pública.

19 Almeida GN, Magalhães RB, Barbosa JB, Hogg T, Teixeira PC: Listeriosis en Portugal: 2004-2007. RPDI 2009;5:90-92.

20 Almeida G, Morvan A, Magalhães R, Santos I, Hogg, T, Leclercq A, et al: Distribution and characterization of Listeria monocytogenes clinical isolates in Portugal, 1994-2007. Eur J Clin Microbiol 2010;30:1219-1227.

21 International Organization for Standardization: Microbiology of Food and Animal Feeding Stuffs: Horizontal Method for the Detection and Enumeration of Listeria monocytogenes: Part 2: Enumeration Method. London, ISO, 1998, pp 11290-11292.
22 International Organization for Standardization: Software and System Engineering: HighLevel Petri Nets: Part 1: Concepts, Definitions and Graphical Notation: Amendment 1: Symmetric Nets. London, ISO, 2004, p 15909.

23 International Organization for Standardization: Microbiology of Food and Animal Feeding Stuffs: Horizontal Method for the Detection and Enumeration of Listeria monocytogenes: Part 1: Detection Method. London, ISO, 1996, p 11290.

24 Santos MI, Correia C, Cunha MI, Saraiva MM, Novais MR: Valores guia para avaliação da qualidade microbiológica de alimentos prontos a comer preparados em estabelecimentos de restauração. ROF 2005;64:66-68.

25 Kamat AS, Nair PM: Identification of Listeria innocua as a biological indicator for inactivation of L. monocytogenes by some meat processing treatments. Lebensm Wiss Technol 1996;29:714-720.

26 Petran RL, Swanson KMJ: Simultaneous growth of Listeria monocytogenes and Listeria innocua. J Food Protect 1993;56:616-618.
27 Arrese E, Arroyo-Izaga M: Prevalence of Listeria monocytogenes in Idiazabal cheese. Nutr Hosp 2012;6:2139-2214.

28 Giammanco GM, Pepe A, Aleo A, D' Agostino V, Milone S, Mammina C: Microbiological quality of Pecorino Siciliano "primosale" cheese on retail sale in the streetmarkets of Palermo, Italy. New Microbiol 2011;34:179-185.

29 Esho FK, Enkhtuya B, Kusumoto A, Kawamoto K: Microbial assessment and prevalence of foodborne pathogens in natural cheeses in Japan. Biomed Res Int 2013;205801.

30 Gould LH, Mungai E, Behravesh CB: Outbreaks attributed to cheese: differences between outbreaks caused by unpasteurized and pasteurized dairy products, United States, 1998-2011. Foodborne Pathog Dis 2014;11: 545-551.

31 U.S. Department of Health and Human Services, Centers for Disease Control and Prevention (CDC): Listeria and Food. Atlanta, CDC, 2013. http://www.cdc.gov/foodsafety/ specificfoods/listeria-and-food.html (accessed January 21, 2015). 